Prog Biophys Mol Biol. 2011 August ; 106(2): 337-339. doi:10.1016/j.pbiomolbio.2011.07.009.

\title{
Systems biology and cancer
}

\author{
Ana M. Soto [Guest editor], \\ Department of Anatomy and Cellular Biology, Tufts University School of Medicine, 136 Harrison \\ Avenue, Boston, MA 02111, USA \\ Carlos Sonnenschein [Guest editor], \\ Department of Anatomy and Cellular Biology, Tufts University School of Medicine, 136 Harrison \\ Avenue, Boston, MA 02111, USA \\ Philip K. Maini [Guest editor], and \\ Centre for Mathematical Biology, Mathematical Institute, 24-29 St Giles', Oxford OX1 3LB, UK. \\ Oxford Centre for Integrative Systems Biology, Department of Biochemistry, South Parks Road, \\ Oxford OX1 3QU, UK \\ Denis Noble* [Editor] \\ Department of Physiology, Anatomy \& Genetics, Parks Road, Oxford OX1 3PT, UK
}

The systems approach to complex biological problems has rapidly gained ground during the first decade of this century. There are several reasons for this development. An important one is that while the achievement of sequencing the complete human genome, and those of other species, has been of great benefit to fundamental science, for example in comparative genomics and evolutionary biology, it has not led to the expected quick and simple solutions to multifactorial diseases (2010). On the contrary, cancer, cardiovascular, respiratory, metabolic and nervous diseases have all been resistant to reductionist analysis. In the case of cancer the hope that by identifying what are called oncogenes we would not only understand cancer but be led naturally to its cure has not been fulfilled (Sonnenschein and Soto, 1999, 2011). In all areas of medical science, despite the identification of hundreds more potential targets by genome sequencing, the pharmaceutical industry has been faced with a decline in the production of new successful drugs. The more we find out about the fundamental elements of biology, the DNA, RNAs, proteins, metabolites, membrane systems, organelles, the more puzzling the picture becomes. Even central biological concepts, like that of a gene, have changed and have even become difficult to define (Beurton et al., 2008).

Reassessment of the fundamental concepts of biological science is therefore necessary. This is happening in all fields, including genetics (Beurton et al., 2008), evolution (Pigliucci and Müller, 2010; Gissis and Jablonka, 2011; Shapiro, 2011), cancer (Soto et al., 2008), development and the relationships between genomes and phenotypes (Noble, 2011b, 2011a). What once were heresies seem to be creeping back into mainstream biology.

\footnotetext{
(C) 2011 Published by Elsevier Ltd.

*Corresponding author. Tel.: +44 1865 272528; fax: +44 1865 272554. denis.noble @ dpag.ox.ac.uk (D. Noble).
} 
One of the driving forces of this development is the use of mathematical modelling in systems biology. This has brought a rigorous quantitative approach to what otherwise would be largely untestable theories. Mathematical models provide a framework in which to interpret the vast amount of experimental data generated on a daily basis and to suggest subsequent experiments necessary to test theories. The traditional verbal reasoning approach is not appropriate in many cases due to the complexity of biology (Gatenby and Maini, 2003) which renders intuition insufficient as results are often counter-intuitive, a characteristic outcome of scientific research that goes as far back as Copernicus' proposal of an heliocentric planetary system. This vast complexity requires a mathematical approach.

The motivation for this focussed issue of the journal is that the field of cancer is ripe for the systems biology approach. As editors we have collected an eclectic mix of articles. This is not a 'one view fits all' approach. It is rather one to 'let a hundred flowers bloom'. At this stage in our understanding we cannot be sure where the next big insights are going to come from.

Since the 18th century biologists and philosophers tried to define the place of biology ${ }^{1}$ in science and in particular its relationship with physics. A two hundred year debate followed, with biologists adopting "physicalist" or "vitalistic" stands. Was life to be explained in a totally materialistic way by the laws of physics? Or were there additional "forces" present in the living matter but absent in the inert one? Curiously, as vitalism dwindled among biologists in the 20th century, physicists like Schrödinger (1944) and Elsasser (1987) were the ones that tried to understand biological order and were prepared to find new laws that applied only to living matter. ${ }^{2}$ No new laws resulted from this search, but from the emerging field of information theories, biologists adopted information as the metaphor for the study of biological organization. ${ }^{3}$ This, however, has not produced the desired effects either, probably because the attempts to formalize this approach failed, which in turn suggests that it was conceptually wrong. Can biology achieve formalization through mathematics, a feat that physics has accomplished so successfully?

The article by Giuseppe Longo and Mael Montevil (2011) (mathematicians), analyzes the principles of intelligibility in physics, which is based on symmetries, and posit that the role of symmetries in biology is different: in their words "the permanent change of symmetries ...per se modifies the analysis of the internal and external processes of life, both in ontogenesis and evolution". They propose to consider the roles played by local and global symmetry changes, along extended critical transitions. According to them, the mathematization of this state of extended criticality may provide the adequate frame to understand biological complexity. Paul-Antoine Miquel (2011) (a philosopher), reflects on the philosophical aspects of the theoretical analysis by Longo and Montevil and concludes

\footnotetext{
${ }^{1}$ The noun Biology was coined later, in 1806 by Lamarck.

${ }^{2}$ We must therefore not be discouraged by the diffficulty of interpreting life by the ordinary laws of physics. For that is just what is to be expected from the knowledge we have gained of the structure of living matter. We must also be prepared to find a new type of physical law prevailing in it. Or are we to term it a non-physical, not to say a super-physical, law? - (Schrödinger, E. What is life? Cambridge University Press, 1944).

${ }^{3}$ This resulted in the ill conceived idea to declare that embryonic development was ruled by a program, and that all the teleological concepts (purposeful design) could now be attributed to programs residing in the DNA... But...only philosophers dared to notice that this substitution had changed nothing: who wrote the program, anyway?
} 
that "the philosophical key point for us is that they (Longo and Montevil) interpret this mathematical space in which anti-entropy is realized in biological criticality as an extension of the classical physical theoretical frameworks." These two contributions aim at improving our understanding on why the principles governing living organisms are different from those defining the physicality of inanimate objects and provide a conceptual frame of reference and a point of departure for constructing a mathematics for biology.

Stuart Baker (a bio-statistician) and Barnett Kramer (a cancer epidemiologist) (2011) evaluate the potential contributions of different approaches to Systems Biology when applied to uncover buried messages in the genesis of cancer which may set new trends in research and in ways to benefit patients. They anticipate both promises and perils in applying systems biology to cancer. The great promise of systems biology comes from the idea that studying a system can provide information not available by separately studying the workings of each part. However, they perceive a divide between systems biology based on the principles of biology or biophysics, systems biology related to statistics, bioinformatics, and reverse engineering, and systems biology involving clinical predictions, sometimes without full appreciation of other viewpoints. The peril comes when the rules leading to a complex system vary over many components and the sample sizes are limited for identifying the rules and making predictions. Baker et al. have introduced the concept of "paradigm instability" when referring to current state of affairs through which the field of cancer research is traversing. Thus, they focus on a number of paradoxes that exist in this field and cautiously point at ways that might increase knowledge about the disease and also benefit patients.

Simon Rosenfeld (2011) (a mathematical physicist) makes a critical analysis of the assumptions and concepts used in the emerging field of network biology, particularly those on the actual physics and chemistry happening inside cells. He posits that, in biology there is dual causality, that is, in addition to the constraints imposed by the laws of nature, there is the evolutionary history of the organism: “...inherent dynamical instability represents the natural laws and physico-chemical principles whereas biological robustness is the result of evolutionary history in which this dynamical instability has been effectively used for gaining evolutionary advantages and survival." He subscribes to the notion that "Mathematics represents a systematic and orderly way of describing and organizing knowledge. In the majority of scientific disciplines, mathematical reasoning has proven to be an unparalleled and indispensable tool for understanding complex dynamics." He forcefully argues for adopting a Systems Biology approach to resolve complex biological problems while complying with a comprehensive evolutionary perspective.

Plankar et al. (2011) challenge the genetically determined paradigm of cancer from another angle to characterise cancer as the result of impaired coherence leading to progressive destabilisation of molecular and gene regulatory networks. As they write in their conclusion "It is becoming clear that even with potentially unlimited insight into the dynamics of genetic changes, cancer could not be sufficiently explained, and neither could it be explained in terms of separate linear molecular pathways alone. During the last decade, scientific attention has turned dramatically towards the metabolic, bioenergetic, developmental, and 
systems biology aspects of cancer, reflecting a gradual paradigm shift towards its nongenetic origin."

Enderling and Hahnfeldt (2011) analyse the dynamics of a growing solid tumour composed of cancer stem cells and cancer non-stem cells using a simple hybrid cellular automaton (CA) model. They illustrate the counter-intuitive finding that increasing the rate of apoptosis, while obviously reducing tumour size in the short-term, actually enhances growth in the long-term. They show that tumours can remain dormant for a long time but stimulation of apoptosis can cause the tumour cell population to aggressively invade. Their work suggests that the widely regarded "evading cell death" as a hallmark of cancer (Hanahan and Weinberg, 2000) needs to be revisited.

Kim et al. (2011) begin by reviewing the interactions between a tumour and its microenvironment, highlighting how this plays an important role in the transition from benign or pre-malignant tumour to invasive cancer. They then describe a continuum model for the mechanics of a growing tumour in three spatial dimensions, and use it to investigate the effects on tumour growth of agarose gel inhomogeneities and other microenvironmental factors. This framework is extended to explore ductal carcinoma in situ (DCIS) in which the stroma is modelled as a continuum but the cells of the tumour are modelled discretely. The mechanical model is coupled to the biochemistry via a system of reaction-diffusion equations which describe the dynamics of key signalling factors. This multiscale model is solved numerically and effects of perturbing the system mechanically or biochemically are illustrated. This approach allows us to begin to understand the outcome of the nonlinear interactions of some of the fundamental processes involved in tumour growth, with the potential to then consider methods to control growth and spread.

Gerlee and Anderson (2011) focus on mechanisms present in organisms that allow it, or parts of it, to maintain a given shape or architecture (structural homeostasis). They consider a hybrid CA model for a two-dimensional mono-layer of cells which may, for example, approximate the epithelial lining of an organ. In their model, each cell has an intracellular network which integrates the cues a cell receives from its microenvironment (for example nutrients or growth factors, whose dynamics are modelled by reaction-diffusion equations) and other cells and determines the response of the cell, in terms of its behaviour or phenotype. The problem is then reduced to finding a set of network parameters (or genotype) which maximises a fitness function such that structural homeostatis is attained. Perturbations of the system, such as wounding or mutation, are investigated.

Vera et al. (2011) present an in-depth review which focuses on JAK-STAT (Janus kinase signal transducer and activator of transcription) pathway in the context of cancer. This pathway plays a fundamental role in growth control, cell differentiation and maintenance of tissue homeostasis, and its dysregulation plays an important role in tumourigenesis. They review the biology of the pathway and then survey systems biology approaches that have helped elucidate the dynamics of the pathway under physiological and diseased states.

Scianna et al., (2011) address the multiple levels of organisation involved in vascularisation, an important step enabling tumour growth and the formation of metastases. Their work 
forms an innovative multiscale hybrid framework within which to test potential antiangiogenic strategies in treating cancer.

Insuk Lee (2011) presents a holistic model of genes as a collaborative society. To the standard approaches involving protein-protein interaction networks (PPIN) and transcriptional regulatory networks (TRN) he adds the probabilistic functional gene network (PFGN) to show how robustness can arise despite noisy genomics data. Mapping epistatic interactions between genes is identified as the key way to understanding the genetic organisation of complex traits. Amongst the applications of this approach he considers epistatic interactions between hub cancer genes such as $\mathrm{p} 53$.

Keith Baverstock (2011) uses models of cell regulation to address the important question of whether regulatory networks are hard wired into the genome or whether they are better represented as open systems involving an attractor interacting with the environment. In the latter case, environmental stress can trigger inherited transitions in the phenotype without necessarily involving DNA sequence changes. The second type of model works best. As he says "the power of the model lies in its ability to make evident how it is that a rigid and highly conserved coding sequence in DNA, the genotype, can give rise to phenotypic plasticity and responsiveness to environment" and that it helps to understand "the origins of non-genetic somatic and inherited disease, arising from switches to variant attractors representing phenotypes with abnormal characteristics." The relevance to diseases like cancer is obvious.

Taken as a whole, this set of articles not only challenges some of the current paradigms, but also lays the groundwork for alternative approaches and in many cases takes those approaches further towards the goal of understanding cancer as a systems-level process.

\section{References}

Baker S, Kramer B. Systems biology and cancer: promises and perils. Progress in Biophysics and Molecular Biology. 2011; 106:411-414.

Baverstock K. A comparison of two cell regulatory models entailing high dimensional attractors representing phenotype. Progress in Biophysics and Molecular Biology. 2011; 106:444-450.

Beurton, PJ.; Falk, R.; Rheinberger, H-J., editors. The Concept of the Gene in Development and Evolution: Historical and Epistemological Perspectives. Cambridge University Press; Cambridge: 2008.

The human genome at ten. Nature. 2010; 464:649-650. Editorial. [PubMed: 20360688]

Elsasser, WM. Reflections on a Theory of Organisms. Orbis; Frelighsburg, Quebec: 1987.

Enderling H, Hahnfeldt P. Cancer stem cells in solid tumors: is "evading apoptosis" a hallmark of cancer? Progress in Biophysics and Molecular Biology. 2011; 106:392-400.

Gatenby RA, Maini PK. Mathematical oncology. Cancer summed up. Nature. 2003; 421:321. [PubMed: 12540881]

Gerlee, Basanta; Anderson, RA. Evolving homeostatic tissue using genetic algorithms. Progress in Biophysics and Molecular Biology. 2011; 106:415-426.

Gissis, SB.; Jablonka, E., editors. From Subtle Fluids to Molecular Biology. MIT Press; Cambridge, Mass: 2011. Transformations of Lamarckism.

Hanahan D, Weinberg RA. The hallmarks of cancer. Cell. 2000; 100:57-70. [PubMed: 10647931]

Kim Y, Stolarska MA, Othmer HG. The role of the microenvironment in tumor growth and invasion. Progress in Biophysics and Molecular Biology. 2011; 106:354-380. 
Lee I. Probabilistic functional gene societies. Progress in Biophysics and Molecular Biology. 2011; 106:436-443.

Longo G, Montevil M. From physics to biology by extending criticality and symmetry breaking. Progress in Biophysics and Molecular Biology. 2011; 106:341-348.

Miquel PA. Extended physics as a theoretical framework for systems biology. Progress in Biophysics and Molecular Biology. 2011; 106:349-353.

Noble D. Differential and integral views of genetics in computational systems biology. Journal of the Royal Society Interface Focus. 2011a; 1:7-15.

Noble D. Neo-Darwinism, the modern synthesis, and selfish genes: are they of use in physiology? Journal of Physiology. 2011b; 589:1007-1015. [PubMed: 21135048]

Pigliucci, M.; Müller, GB., editors. Evolution - The extended synthesis. MIT Press; Cambridge, Mass: 2010.

Plankar M, Jerman I, Krasovic R. On the origin of cancer: can we ignore coherence? Progress in Biophysics and Molecular Biology. 2011; 106:381-391.

Rosenfeld S. Mathematical descriptions of biochemical networks: stability, stochasticity, evolution. Progress in Biophysics and Molecular Biology. 2011; 106:401-410.

Schrödinger, E. What is Life?. Cambridge University Press; Cambridge: 1944.

Scianna M, Munaron L, Preziosi L. A multuscale hybrid approach for vasculogenesis and related potential blocking therapies. Progress in Biophysics and Molecular Biology. 2011; 106:451-463.

Shapiro, JA. Evolution: A View from the 21st Century. Pearson Education Inc; Upper Saddle River, NJ: 2011.

Sonnenschein, C.; Soto, A. The Society of Cells: Cancer and Control of Cell Proliferation. Taylor \& Francis Group; Garland: 1999.

Sonnenschein C, Soto A. The death of the cancer cell. Cancer Research. 2011; 71:4334-4337. [PubMed: 21507929]

Soto AM, Sonnenschein C, Miquel PA. On physicalism and downward causation in developmental and cancer biology. Acta Biotheoretica. 2008; 56:257-274. [PubMed: 18542843]

Vera J, Rateitschaka K, Langea F, Kossowa C, Wolkenhauer O, Jaster R. Systems biology approach on JAK-STAT signalling in human malignancies. Progress in Biophysics and Molecular Biology. 2011; 106:427-435. 